\title{
La calciphylaxie et ses manifestations stomatologiques
}

\section{Calciphylaxis and its stomatologic manifestations}

\author{
TATJANA ZAGURY-CHAPPUIS, JEAN-PIERRE CARREL, SEMAAN ABI NAJM, JACKY SAMSON
}

\begin{abstract}
RÉSUMÉ
L'anamnèse et l'examen clinique permettent parfois de découvrir des affections locales ou systémiques interférant avec le traitement envisagé. Quelquefois, le praticien se trouve confronté directement à une affection médicale. Dans tous les cas, il ne doit pas se limiter à un examen buccal sommaire mais s'efforcer de réaliser une prise en charge holistique. La calciphylaxie constitue un exemple caricatural, mais démonstratif pour illustrer cette démarche car les manifestations stomatologiques sont exceptionnelles. Cette maladie rare, multifactorielle, potentiellement fatale, est surtout rencontrée chez des patients atteints d'une insuffisance rénale chronique. Elle se caractérise par une calcification des artères avec apparition de nécroses ischémiques périphériques. Une meilleure connaissance des différentes hypothèses étiopathogéniques, des troubles métaboliques observés et des facteurs de risque permettraient de la dépister plus précocement et de proposer aux patients un traitement préventif adéquat.

La présentation d'un cas de calciphylaxie avec des manifestations stomatologiques initiales montre clairement que le diagnostic aurait pu être évoqué plus précocement si on avait transposé, à la région cervicofaciale, les manifestations de la calciphylaxie observées habituellement aux extrémités. (Med Buccale Chir Buccale 2006; 12: 89-99).
\end{abstract}

médecine buccale chirurgie buccale VOL. $12, \mathrm{~N}^{\circ} 2$ 2006 page 89

mots clés : calciphylaxie, insuffisance rénale chronique, manifestations stomatologiques

\section{SUMMARY}

Complete medical history and full clinical examination of a patient may lead to the discovery of local or systemic affections interfering with a dental treatment. To illustrate the importance of this, calciphylaxis is a good example because oral signs are rare and appear late. It is an uncommon disease, potentally fatal in patients with chronic renal insufficiency, characterized by vascular calcifications leading to peripheral ischaemical necrosis. A good knowledge of metabolic disorders, etiopathogenesis and risk factors of this multifactorial disease is essential for early recognition and adequate preventive measure. The present case demonstrates that the diagnosis could have been evoked earlier if the clinical signs of calciphylaxis in the cervicofacial region had been regognized. (Med Buccale Chir Buccale 2006; 12: 89-99).

key words: calciphylaxis, chronic renal insufficiency, stomatological lesions

* Division de Stomatologie, Chirurgie Orale et Radiologie Dento-Maxillo-Faciale, Ecole de Médecine Dentaire, Genève

Demande de tirés à part :

Tatajna Zagury-Chappuis Division de Stomatologie Chirurgie Orale et Radiologie Dento-Maxillo-Faciale Ecole de Médecine Dentaire

Rue Barthélemy-Menn 191205 Genève Tatjana.Zagury@medecine.unige.ch 
médecine buccale chirurgie buccale

VOL. $12, \mathrm{~N}^{\circ} 2$ 2006

page 90
En odontostomatologie, l'anamnèse et l'examen clinique constituent les étapes initiales à toute prise en charge permettant de découvrir parfois des affections locales ou systémiques interférant avec le plan de traitement envisagé. Parfois, le praticien se trouve confronté directement à une affection médicale. Dans tous les cas, il ne doit pas se limiter à un examen sommaire buccal mais s'efforcer de réaliser une prise en charge holistique. Certes, il ne connaît pas toujours suffisamment la pathologie médicale pour proposer des investigations ou un traitement mais il doit être capable de conseiller et d'orienter le patient, et surtout de contacter le médecin traitant dès que la pathologie semble dépasser le cadre de l'odontostomatologie. La calciphylaxie constitue sans doute un exemple caricatural car les manifestations stomatologiques sont exceptionnelles et presque toujours au second plan. L'analyse du cas présenté montre clairement que le diagnostic aurait pu être évoqué bien plus tôt si on avait transposé, à la région cervico-faciale, les manifestations classiques de la calciphylaxie.

\section{OBSERVATION}

Un homme, né en 1941, présente une maladie de Von Willebrand, une polykystose rénale bilatérale et une hypertension artérielle maligne ; ces deux dernières affections ont favorisé le développement d'une insuffisance rénale chronique (IRC). A partir de 1980, il est traité par hémodialyse et, dès 1983, par dialyse péritonéale poursuivie jusqu'au $3^{\mathrm{e}}$ épisode de péritonite en 1989. Entre 1980 et 1984, il y a eu deux greffes rénales qui ont été chaque fois suivies d'un rejet.

En juillet 1985, il consulte dans la Division de Stomatologie et Chirurgie orale pour une lésion kératosique verruqueuse de la joue gauche, développée sur un état post-lichénien, qui ne présente aucun signe de malignité. L'apparition de douleurs amène le patient à consulter de nouveau 6 mois plus tard. L'examen clinique fait suspecter une transformation maligne, confirmée par l'examen histopathologique : il s'agit d'une papillomatose inversée comportant un petit foyer de carcinome épidermoïde. L'exérèse est effectuée en janvier 1986 mais une récidive survient en juin 1986. L'orthopantomogramme, réalisé lors du bilan pré-opératoire, ne montre aucune anomalie (Fig. 1). Une intervention chirurgicale comportant une exérèse tumorale avec résection interruptrice de la mandibule et un évidement ganglionnaire radical homolatéral, est réalisée en juin 1986. Des métastases sont retrouvées dans 7 ganglions, mais aucun ne comporte une rupture capsulaire. Le traitement chirurgical est complété par une radiothérapie sur les aires ganglionnaires cervico-faciales. En septembre 1986, le patient est hospitalisé dans le service de Dermatologie de l'Hôpital Cantonal de Genève pour une infection herpétique buccale étendue qui a nécessité un traitement par l'aciclovir (250 mg, x 2. $\left.\mathrm{j}^{-1}\right)$.

En 1989, le $3^{e}$ épisode de péritonite s'est compliqué d'une septicémie ayant nécessité 3 mois d'hospitalisation. Pendant son séjour hospitalier, le patient a abandonné la fluoration topique de ses dents. Dans les mois qui ont suivi, il a développé des caries sur toutes ses dents : elles ont dû être toutes extraites, sauf la 43, entre septembre 1991 et janvier 1992.

En octobre 1990, des douleurs de type claudication intermittente, ressenties régulièrement depuis quelques mois pendant la mastication et la déglutition, deviennent plus vives et ont tendance à persister entre les repas. Elles intéressent surtout la langue mais aussi probablement les muscles pharyngés et peut-être certains muscles masticateurs ; dans un premier temps, ces dernières sont assimilées à des irradiations de la douleur linguale qui est

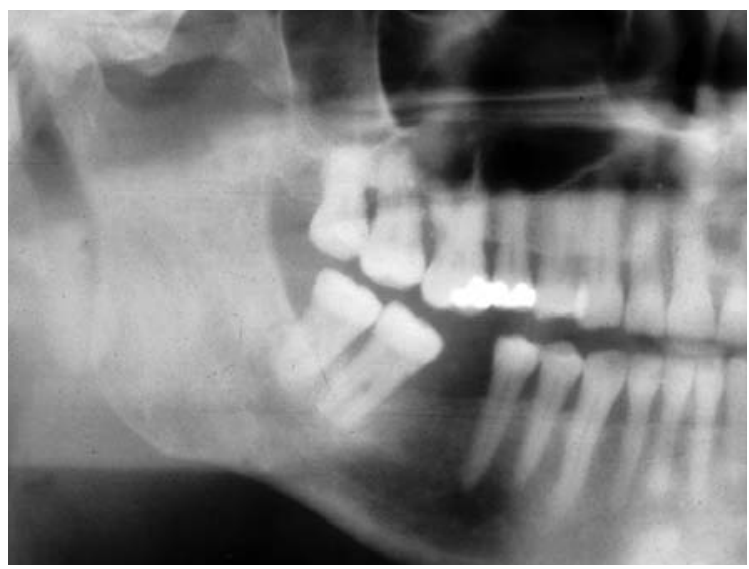

Figure 1 : Détail de la partie droite de l'orthopantomogramme de 1986.

Detail of the right part of the orthopantomogramme done in 1986. 




Figure 2 : Zone nécrotique intéressant l'hémilangue droite dont le fond est recouvert d'un enduit fibrinoleucocytaire et des tissus nécrosés. Les reflets bleus sont dus à l'application de bleu de méthylène (décembre 1991).

Necrosis of right side of the tongue covered by a fibrin slough and necrotic tissues. The staining is due to methylene blue (december 1991).

considérée comme une séquelle de la radiothérapie. Leur intensité devient telle qu'elle interdit toute alimentation par voie buccale, impose la prescription de morphine et la mise en place d'une sonde naso-gastrique. L'artériographie, réalisée fin novembre 1990, montre des lésions sténosantes touchant les branches terminales de l'artère faciale droite; cette dernière et la carotide externe homolatérale ont un diamètre normal. Début décembre 1991, il apparaît une vaste zone nécrotique $(5 \times 2 \mathrm{~cm})$ intéressant le bord droit de la langue et la face dorsale avoisinante (Fig. 2). En l'absence de diagnostic étiologique et devant l'ignorance des

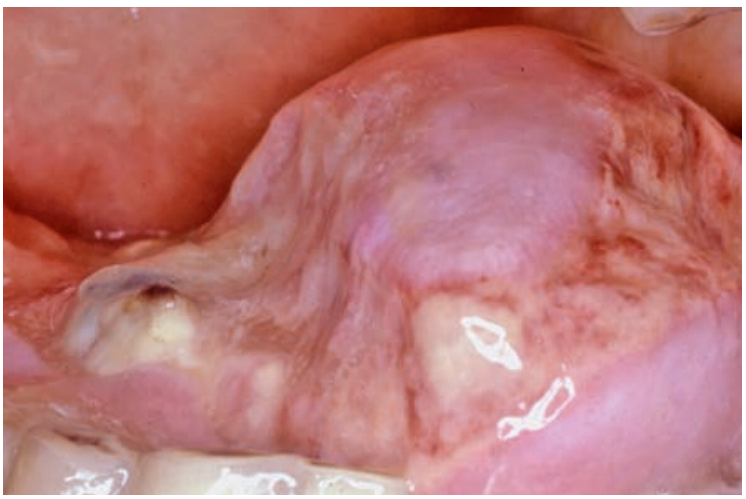

Figure 3 : Détersion de la zone nécrotique de l'hémilangue droite et apparition d'une zone nécrotique plus superficielle et de taille réduite sur l'hémilangue gauche. Necrosis of the right side of the tongue with the presence of a superficial and smaller necrotic area on the left side of the tongue.

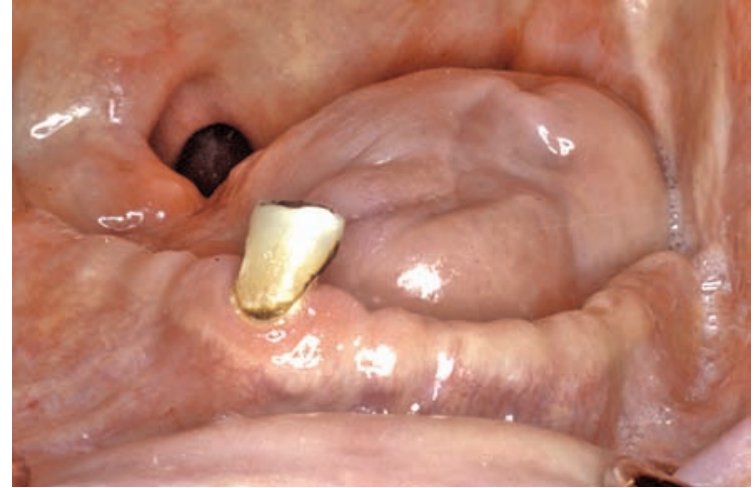

Figure 4 : Cicatrisation des deux zones nécrotiques se traduisant par l'amputation partielle de la langue (fin janvier 1992).

Healing of both necrotic areas resulted in a partial amputation of the tongue (end of january 1992)

limites exactes de la nécrose, une attitude conservatrice est adoptée. L'évolution, lentement favorable, va durer plusieurs semaines; pendant ce temps, une petite zone nécrotique est apparue sur le bord gauche de la langue (Fig. 3). Les soins locaux (désinfection et élimination du tissu nécrotique dès son apparition) et une antibiothérapie ont permis d'obtenir une cicatrisation par seconde intention, respectivement en 2 mois et 3 semaines (Fig. 4). Sur les orthopantomogrammes réalisés en janvier 1991 (Fig. 5) et en septembre 1991 (Fig. 6), l'artère faciale droite est calcifiée depuis son origine jusque dans la région du bord basilaire de la mandibule. Elle est facilement perçue à la palpation sous la forme d'un cordon dur, non pulsatile. Le diagnostic d'ischémie post-actinique ou de maladie de Horton, évoqué initialement, est abandonné devant l'apparition de douleurs touchant les $2^{e}$ et $3^{e}$ orteils gauches, associées à une insuffisance artérielle du membre inférieur gauche (stade IV). Malgré la réalisation d'un pontage fémoro-poplité (janvier 1991), les douleurs persistent et des ulcérations nécrotiques apparaissent sur les $2^{\mathrm{e}}$ et $3^{\mathrm{e}}$ orteils gauches (Fig. 7), associées à un œdème malléolaire. Afin de faciliter l'alimentation du patient, une gastrostomie par voie endoscopique est réalisée en juin 1991. Les douleurs s'étendent et touchent maintenant les membres inférieurs et les doigts (janvier1992) (Fig. 8) ; elles sont assez bien contrôlées par la buprénorphine. L'évolution (Fig. 9) oblige à pratiquer l'amputation, sous le genou, de la jambe droite (mars 1992), puis de la jambe gauche (sep- médecine

buccale chirurgie buccale

VOL. $12, \mathrm{~N}^{\circ} 2$ 2006

page 91 


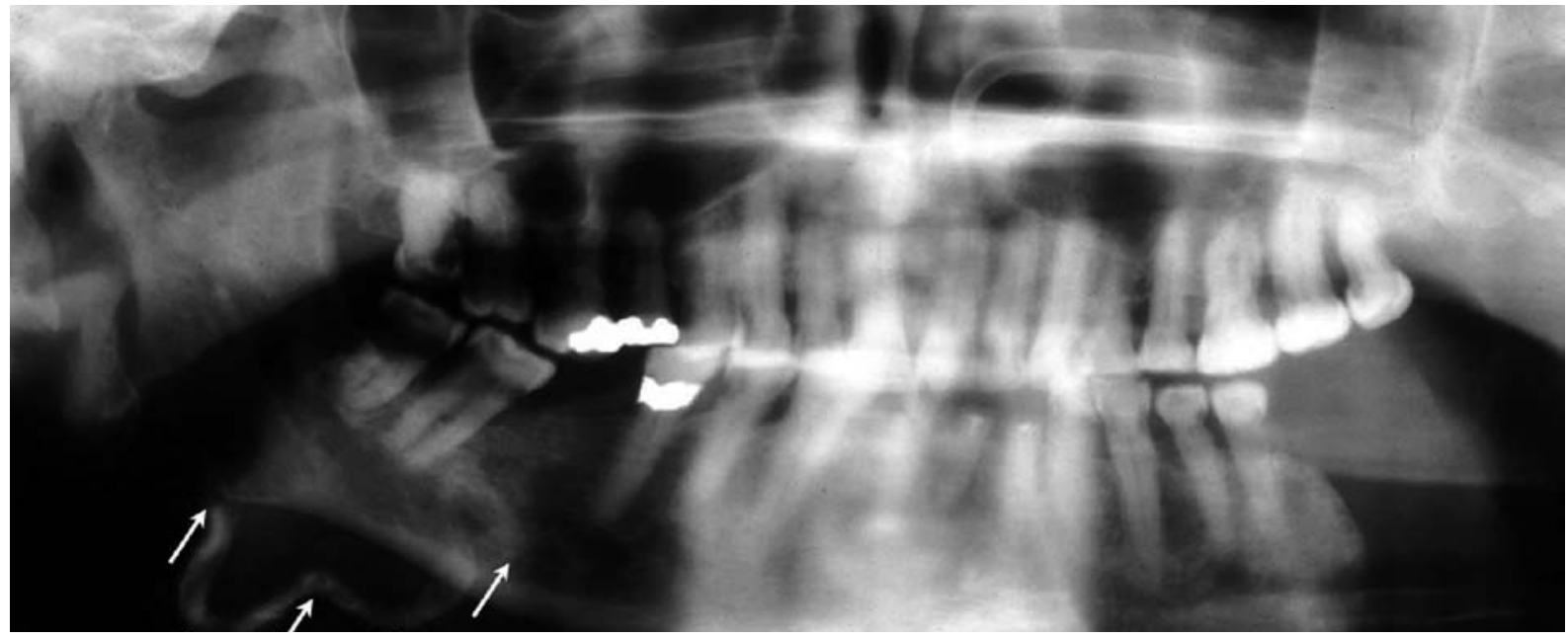

Figure 5 : Orthopantomogramme du 19.01.1991: la calcification de l'artère faciale droite est déjà bien visible. 19.01.1991: Orthopantomogram showing the calcification of the right facial artery.

médecine

buccale

chirurgie

buccale

VOL. $12, \mathrm{~N}^{\circ} 2$ 2006

page 92

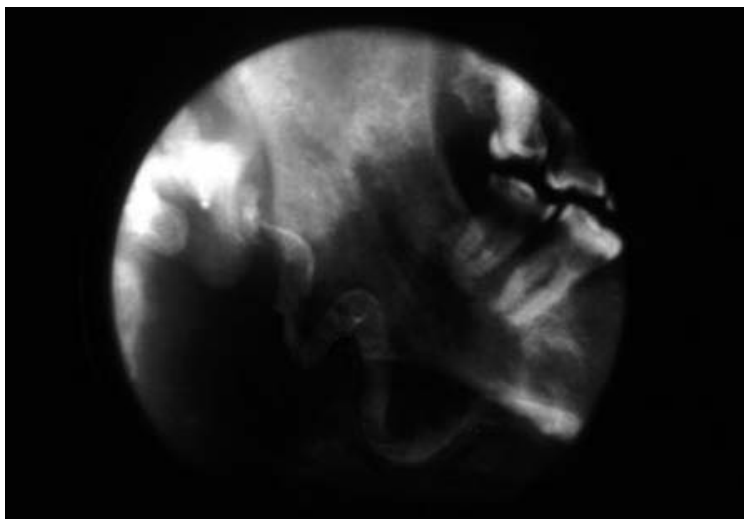

Figure 6 : Détail de l'orthopantomogramme du 19.09.1991: l'artère faciale est encore plus calcifiée. 19.09.1991: Detail of the orthopantomogram with the facial artery more calcified

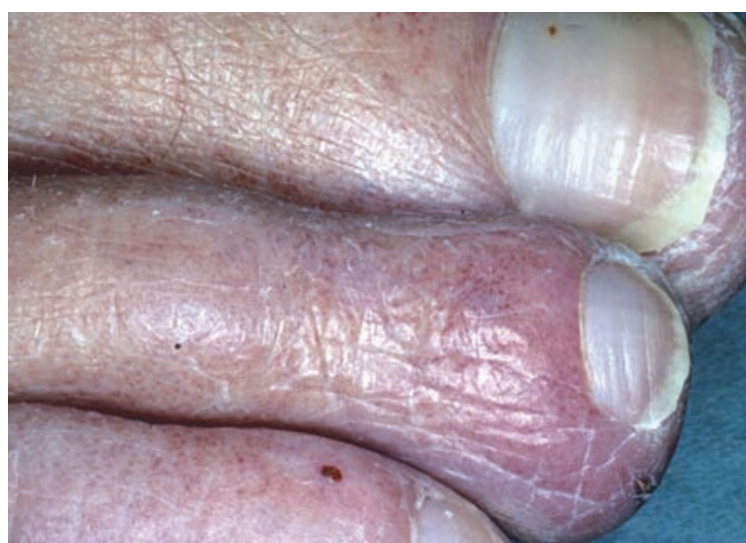

Figure 8 : Erythème de l'extrémité distale du $2^{\ominus}$ orteil droit, principalement dans la région péri-unguéale (janvier 1992).

Erythema of distal part of the second right toe (january 1992), invoving mainly the perinail area.

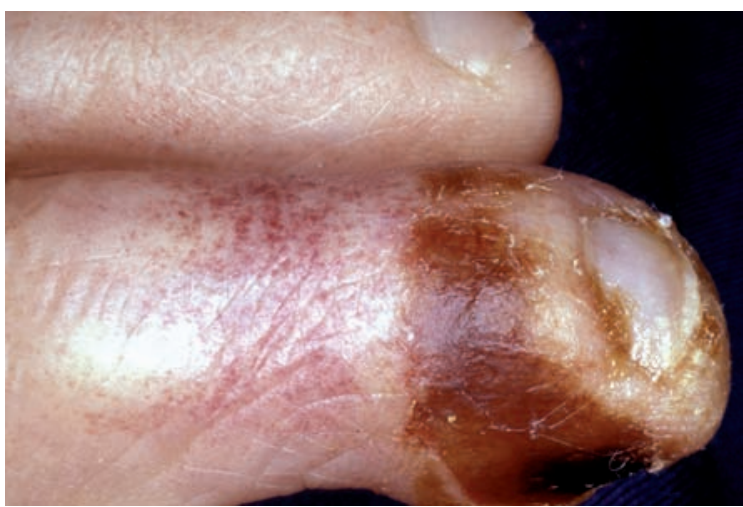

Figure 7 : Ulcération du $2^{e}$ et $3^{\circ}$ orteils gauches (janvier 1991).

Ulcerations of the second and third left toes (january 1991).

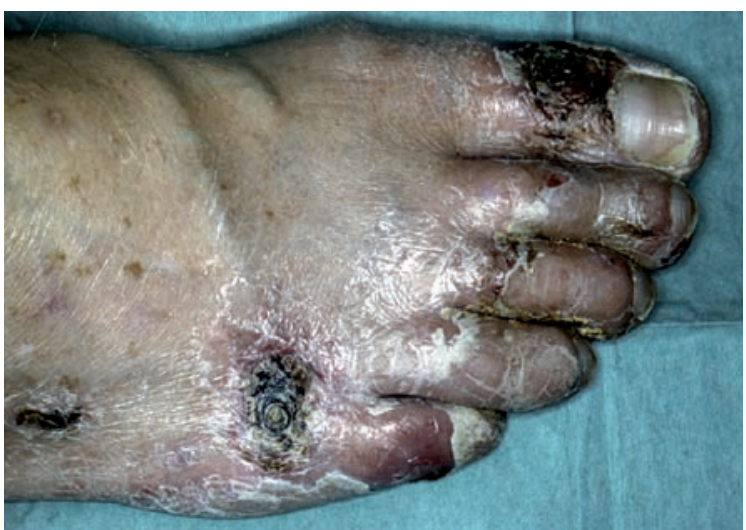

Figure 9 : Nécrose de tous les orteils avec deux zones cutanées nécrotiques sur le pied droit conduisant à son amputation (mars 1992).

Necrosis of all toes with two necrotic areas on the right foot leading to final amputation (march 1992). 
tembre 1993). Des ulcérations nécrotiques se développent sur deux doigts de la main droite (sur l'auriculaire en janvier 1993 - il sera amputé en juillet 1993 - et sur l'index en juillet 1993) et sur le gland en septembre 1993 (amputation du pénis en octobre 1993). Fin novembre 1993, des signes d'ischémie aiguë apparaissent sur le moignon de la jambe droite. L'amputation à mi-cuisse est réalisée début décembre 1993 ; dans le même temps, on pratique l'amputation du pouce et de l'annulaire droits. Après discussion avec le patient et sa famille, les séances d'hémodialyse sont interrompues après Noël et le patient décède le 30 décembre 1993.

\section{COMMENTAIRES}

\section{Calciphylaxie}

\section{Mécanismes physiopathologiques}

En 1855, Virchow a observé, dans I'IRC, la présence de dépôts de calcium dans de nombreux tissus comme la peau, les muscles, les tissus périarticulaires, les artères et les tubules rénaux. Ces dépôts étaient accompagnés d'altérations vasculaires d'évolution progressive et d'arythmies cardiaques ${ }^{[1,2]}$.

En 1898, Bryant et White décrivent pour la première fois la calciphylaxie comme un syndrome rare, comportant des calcifications associées à des nécroses ischémiques périphériques ${ }^{[2,3]}$. Mais C'est à travers le modèle expérimental de Selye ${ }^{[4]}$, utilisant un milieu physiologique comparable à celui de I'IRC au stade terminal, que la plupart des concepts faisant intervenir des facteurs prédisposants pour le développement de la calciphylaxie ont été étudiés en 1962. La calciphylaxie est alors décrite comme une calcification locale aiguë, secondaire à une réaction d'hypersensibilité favorisée par un traitement topique ou par des facteurs sensibilisants ${ }^{[4]}$.

Initialement, les lésions sont caractérisées par des dépôts calciques sous l'intima des artères de petit et moyen calibre, entraînant un rétrécissement de la lumière vasculaire ${ }^{[5-8]}$. L'aspect histopathologique des lésions cutanées n'est pas spécifique de la calciphylaxie car il peut être observé dans de nombreuses maladies comportant une nécrose cutanée spontanée ${ }^{[8]}$. II semble que la calciphylaxie se développe initialement dans la lame élastique interne et qu'elle gagne ensuite la média ; ce n'est que lorsque la lame élastique interne est détruite que la fibrose touche l'intima. On note également la présence d'un infiltrat inflammatoire chronique périvasculaire et sous-cutané diffus ${ }^{[9 .}$. Les calcifications précèdent la fibrose et il y a de nombreux aspects évolutifs intermédiaires. L'absence de cristaux de cholestérol différencie la calciphylaxie de l'artériosclérose. La microradiologie, réalisée en complément aux examens de routine (taux sérique du calcium, de phosphates, de phosphatases alcalines et de la PTH), permet de dépister la calciphylaxie au stade initial, bien avant l'apparition des nécroses cutanées.

\section{Manifestations cliniques}

La prévalence de la calciphylaxie varie de $1 \%$ à $4,1 \%$ chez les patients hémodialysés ${ }^{[10-12]}$. Le sex ratio $\mathrm{M} / \mathrm{F}$ est de $1: 3^{[13,14]}$.

\section{Manifestations cutanéo-muqueuses}

Classiquement, au stade initial, les lésions cutanées sont constituées par des plages violacées rappelant le livedo reticularis ; elles sont accompagnées d'un prurit et elles vont évoluer vers une nécrose superficielle avec formation d'une escarre. Elles sont habituellement bilatérales et symétriques ${ }^{[15,16]}$. Elles touchent principalement les bras, les doigts, la moitié inférieure de la paroi abdominale, les cuisses, les fesses, les mollets, les talons et les orteils. Elles n'ont aucune tendance à la guérison spontanée et, en raison de l'ischémie sous-jacente, la surinfection est difficilement contrôlable et peut engendrer une septicémie. Elles sont accompagnées de douleurs dont l'intensité semble en général disproportionnée par rapport à l'aspect des lésions $[8,15,17]$.

\section{Autres manifestations}

Le système cardiovasculaire peut comporter d'autres atteintes en dehors de celle des artères de petit et moyen calibre; on observe souvent des calcifications intracardiaques et valvulaires mais elles se traduisent rarement par des manifestations cardiaques ou une atteinte de la valve aortique et des gros troncs. II peut exister également un déficit en protéines $C$ et $S$ révélé par le \begin{tabular}{l}
$\begin{array}{l}\text { médecine } \\
\text { buccale } \\
\text { chirurgie } \\
\text { buccale }\end{array}$ \\
\hline VOL. $12, N^{\circ} 2$ \\
2006 \\
\hline page 93
\end{tabular} 
médecine

buccale

chirurgie

buccale

VOL. $12, \mathrm{~N}^{\circ} 2$ 2006

page 94 développement paradoxal d'une thrombose lors de la prescription d'antivitamines $\mathrm{K}$.

L'atteinte musculaire peut se traduire par une myopathie ou une rhabdomyosite, dues à une lésion des sarcolemmes des muscles striés squelettiques [58] ; elle touche principalement les muscles intercostaux, le muscle pectoral, le deltoïde, le psoas-iliaque, le quadriceps et le tibial antérieur. Elle peut, dans de rares cas, précéder les lésions cutanées.

Les patients sous dialyse péritonéale développent parfois une péritonite sclérosante calcifiante dont l'étiopathogénie reste incertaine même si l'infection semble jouer un rôle déterminant.

\section{Manifestations stomatologiques}

Les manifestations cliniques de la calciphylaxie intéressant la cavité buccale sont peu fréquentes et généralement absentes au stade initial. Mathur et al. [18] ont rapporté un cas comportant des nécroses cutanées intéressant la face, qui constitue une localisation rare. Bedoya et al. [1] ont montré qu'exceptionnellement, les manifestations stomatologiques peuvent précéder toutes les autres. Ils ont présenté l'observation d'un patient de 43 ans en IRC, atteint de calciphylaxie, avec des lésions touchant la cavité buccale. L'hémilangue gauche est devenue subitement très douloureuse et violacée. Après 48 heures, elle s'est nécrosée, donnant naissance à une hémorragie importante qui a conduit à réaliser une hémiglossectomie avec ligature de la carotide externe.

\section{Evolution}

Elle est souvent fatale : le taux de mortalité est d'environ $60 \%$ et le décès résulte le plus souvent d'une septicémie. Le taux de mortalité semble supérieur chez les patients atteints de lésions proximales. Hafner et al. [19] retrouvent $63 \%$ de décès chez les patients ayant des lésions proximales et $23 \%$ seulement chez les patients ayant des lésions distales. Ils démontrent également qu'il y a une amélioration chez $65 \%$ des patients après parathyroïdectomie et chez seulement $35 \%$ des patients qui n'ont pas eu de parathyroïdectomie [19]. Dans l'étude de Fine et Zacharias [20], le taux de mortalité s'élève à $33 \%$ chez les patients qui ne présentent pas encore de nécroses cuta- nées ischémiques et à $80 \%$ lorsque les ulcérations sont apparues.

\section{Hypothèses étiopathogéniques}

L'étiopathogénie de la calciphylaxie reste incertaine bien que la théorie de Selye faisant intervenir un agent sensibilisant soit généralement acceptée ${ }^{[4,617]}$. La physiopathologie des différentes interactions entre agents sensibilisants et agents déclenchants conduisant à des dépôts de calcium n'est pas réellement élucidée. Devant des manifestations cutanées caractéristiques, le diagnostic de calciphylaxie est évoqué, même si la concentration sérique du calcium, des phosphates ou de la PTH est normale ou en l'absence d'une IRC sévère [3,21], car l'apparition des lésions ne semble pas être obligatoirement liée à la sévérité de l'IRC [7]. Toutefois, la calciphylaxie est le plus souvent retrouvée chez des patients souffrant d'une IRC au stade terminal, avec une fréquence qui semble avoir augmenté lors de la dernière décennie ${ }^{[1,22]}$.

De nombreux facteurs ont été évoqués ${ }^{[10,17,23]}$ : l'hyperparathyroïdie, un rapport calcium/phosphates anormalement élevé, l'injection d'albumine, un traitement par corticostéroïdes ou immunosuppresseurs, un déficit qualitatif en protéine $C$, un déficit quantitatif en protéine $S$, la présence d'anticoagulants circulants en relation avec une maladie auto-immune, un trouble du métabolisme de l'acide oxalique...

Mazhar et al. ${ }^{[24]}$ ont mis en évidence des facteurs de risque variant selon le sexe et la race du sujet. D'autres facteurs de risque tels un taux d'albumine sérique faible, une concentration élevée des phosphatases alcalines ou une hyperphosphatémie favoriseraient aussi le développement de la calciphylaxie ${ }^{[12,21,24]}$. Maintenant, on ne fait plus de différence entre les facteurs déclenchants et prédisposants étudiés dans le modèle de Selye : ils sont considérés comme des facteurs de risque pour le développement de la calciphylaxie.

En résumé, les facteurs de risque sont nombreux et semblent être les suivants $[10,15,24,25]$ : sexe féminin, origine caucasienne, obésité, IRC, taux de Ca et de $\mathrm{P}$ élevé, taux d'albumine sérique bas, concentration élevée en phosphatases alcalines, corticostéroïdes, médicaments cytotoxiques et 
immunosupresseurs, surcharge en fer, diabète. Le développement de la calciphylaxie peut être considéré comme un processus se déroulant en deux étapes, avec des facteurs de risque intervenant à des niveaux différents (Tab. 1).

\section{Diagnostic différentiel}

II n'existe pas d'examens spécifiques permettant de poser avec certitude le diagnostic de calciphylaxie. L'observation de lésions bilatérales et symétriques, la présence d'une douleur intense ainsi qu'une nécrose superficielle avec persistance des pouls périphériques, superficiel et profond, constituent des signes cliniques importants qui permettent, avec la biopsie cutanée, de confirmer le diagnostic de calciphylaxie ${ }^{[15,26]}$.

Il est nécessaire de différencier la calciphylaxie des maladies vasculaires périphériques, de l'embolie au choléstérol, de l'endocardite marantique, d'une cryoglobulinémie, d'une sclérodermie, de la maladie de Mönckeberg, de la maladie de Horton, de l'hyperoxalurie primaire de type $1 . .$. D'autre part, on doit évoquer les nombreuses nécroses périphériques : l'artérite des membres inférieurs, la sclérodermie, le groupement CREST (Calcinosis, Raynaud's phenomenon, Esophageal dysfonction, Sclerodactyly and Telangiectasis), le lupus érythémateux, la périartérite noueuse, déficit en protéines $\mathrm{C}$, la thrombopénie induite par l'héparine...
Enfin dans le diagnostic différentiel, on doit envisager d'autres affections susceptibles de comporter des ulcérations cutanées (Tab. 2).

Le diagnostic différentiel repose donc sur le tableau clinique, la biopsie cutanée, le taux sérique du calcium, des phosphates, de la PTH ainsi que sur l'appartenance à la population présentant le risque de développer une calciphylaxie, principalement les patients en IRC.

\section{Traitement}

Le traitement de la calciphylaxie est souvent peu satisfaisant, controversé et, pour être efficace, il doit être précoce. Le développement de la calciphylaxie dans I'IRC dépend de nombreux facteurs. Plusieurs approches thérapeutiques ont été proposées mais elles sont toutes limitées par l'IRC [9]. En pratique, il y a deux grandes options thérapeutiques : la parathyroïdectomie ${ }^{[12,27]}$ et l'amputation chirurgicale du(es) membre(s) atteint(s), suivie de soins post-opératoires attentifs ${ }^{[24]}$. Le dépistage précoce est essentiel pour la prévention et l'amélioration du pronostic de cette affection.

II faut contrôler la balance électrolytique et le processus infectieux en lavant et en désinfectant les lésions cutanées. Il est important d'avoir un apport nutritionnel adapté, de traiter les désordres digestifs (ulcère gastrique, pancréatite...) et de surveiller le rythme cardiaque ${ }^{[16,28]}$. Des investigations doivent être réalisées afin de mettre en évidence

Tableau 1 : Facteurs de risque pour le développement de la calciphylaxie [10] Predisposing factors for the developpment of the calciphylaxis

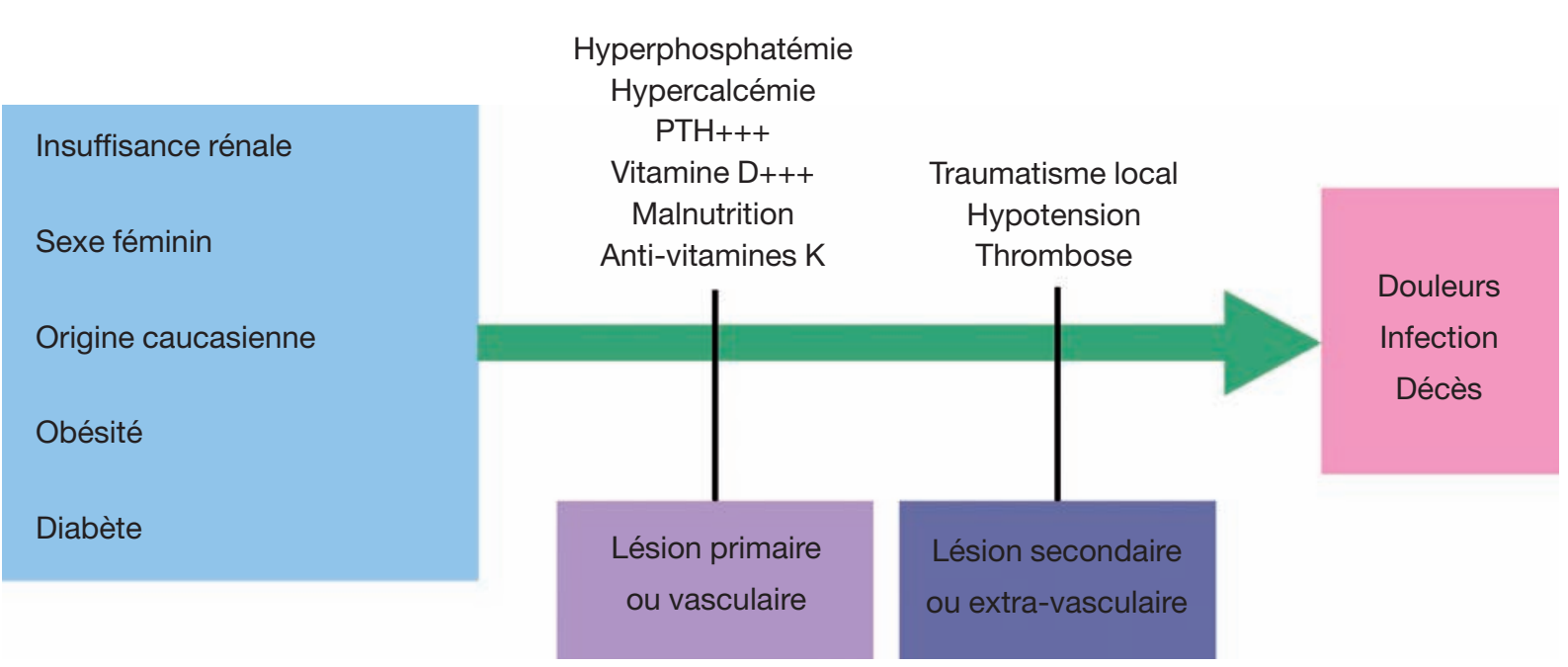


Tableau 2 : Autres affections pouvant comporter des ulcérations cutanées (modifiée d'après ${ }^{[31]}$ ) Others pathological conditions accompanied by cutaneous ulcerations

Affections
Vascularite systémique
Thrombose induite par l'héparine
Nécrose cutanée due aux antivitamines K
Coagulation intravasculaire disséminée
Syndrome des anticorps antiphospholipides
Cryofibrinogénémie
Panniculite pancréatique
Fasciite nécrosante
Embolie infectieuse

Pathogénie/diagnostic
Nécrose fibrinoide
Thrombose microvasculaire,
anticorps responsables de la thrombopénie
induite par l'héparine (Ac TIH)
Thrombose microvasculaire
Thrombose microvasculaire
Thrombose microvasculaire
Vasculite purpurique
Panniculite, amylase, douleurs abdominales
Nécrose des fascias
(streptococcus A-B hémolytique)
Endocardite bactérienne
Dermatose neutrophile, papulopustules,
folliculites ulcérantes

Tableau 3 : Taux de survie des patients traités par parathyroïdectomie et de ceux ayant reçu seulement un traitement médical

Survival rate of patients treated by parathyroidectomy versus patients treated without surgical treatment

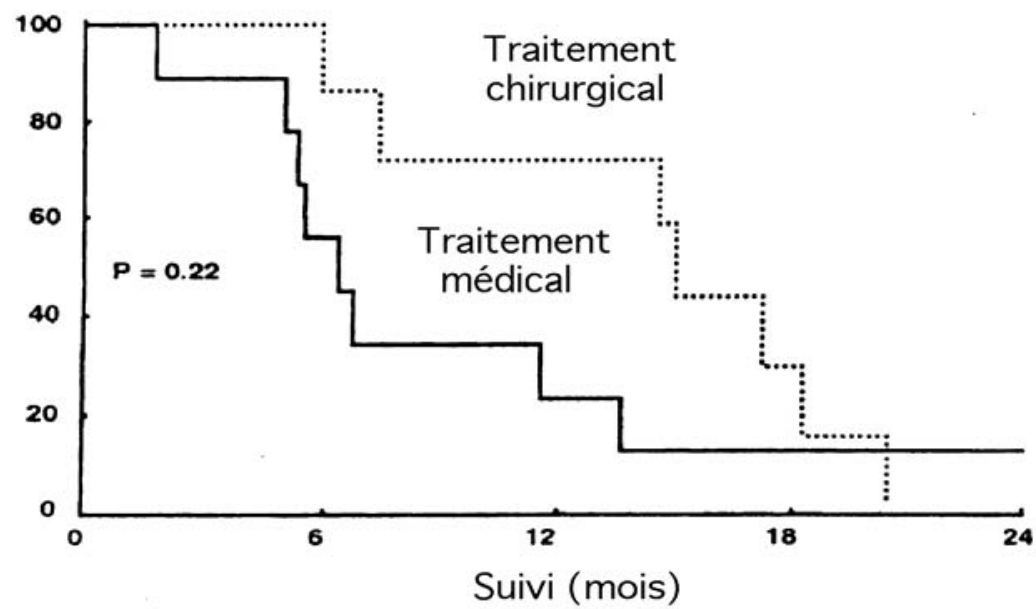


le(s) facteur(s) à risque mais la relation de cause à effet n'est pas toujours facile à établir.

Le résultat de la parathyroïdectomie est d'autant meilleur que le diagnostic et la prise en charge sont précoces, que l'état de santé général du patient est conservé et qu'il n'y a pas d'anomalies métaboliques associées. Le nombre, la distribution et la sévérité des lésions sont également déterminants ${ }^{[15]}$. Hafner et al. [22] ont démontré qu'il y a une amélioration chez $70 \%$ des patients parathyroïdectomisés.
L'efficacité de l'oxygénothérapie pour le traitement des lésions cutanées a également été testé en particulier en présence d'une hyperparathyroïdie secondaire incontrôlable mais les résultats sont peu probants ${ }^{[1]}$.

Une approche multidisciplinaire et une collaboration entre chirurgien, endocrinologue, dermatologue néphrologue, et éventuellement odontostomatologiste, est capitale dans la prise en charge des patients atteints de calciphylaxie (Tab. 4) [29,30].

Tableau 4 : Algorithme pour le traitement de la calciphylaxie [30]. (MVP : maladie vasculaire périphérique) Algorithm for calciphylaxis treatment (MVP: vascular peripheral disease)

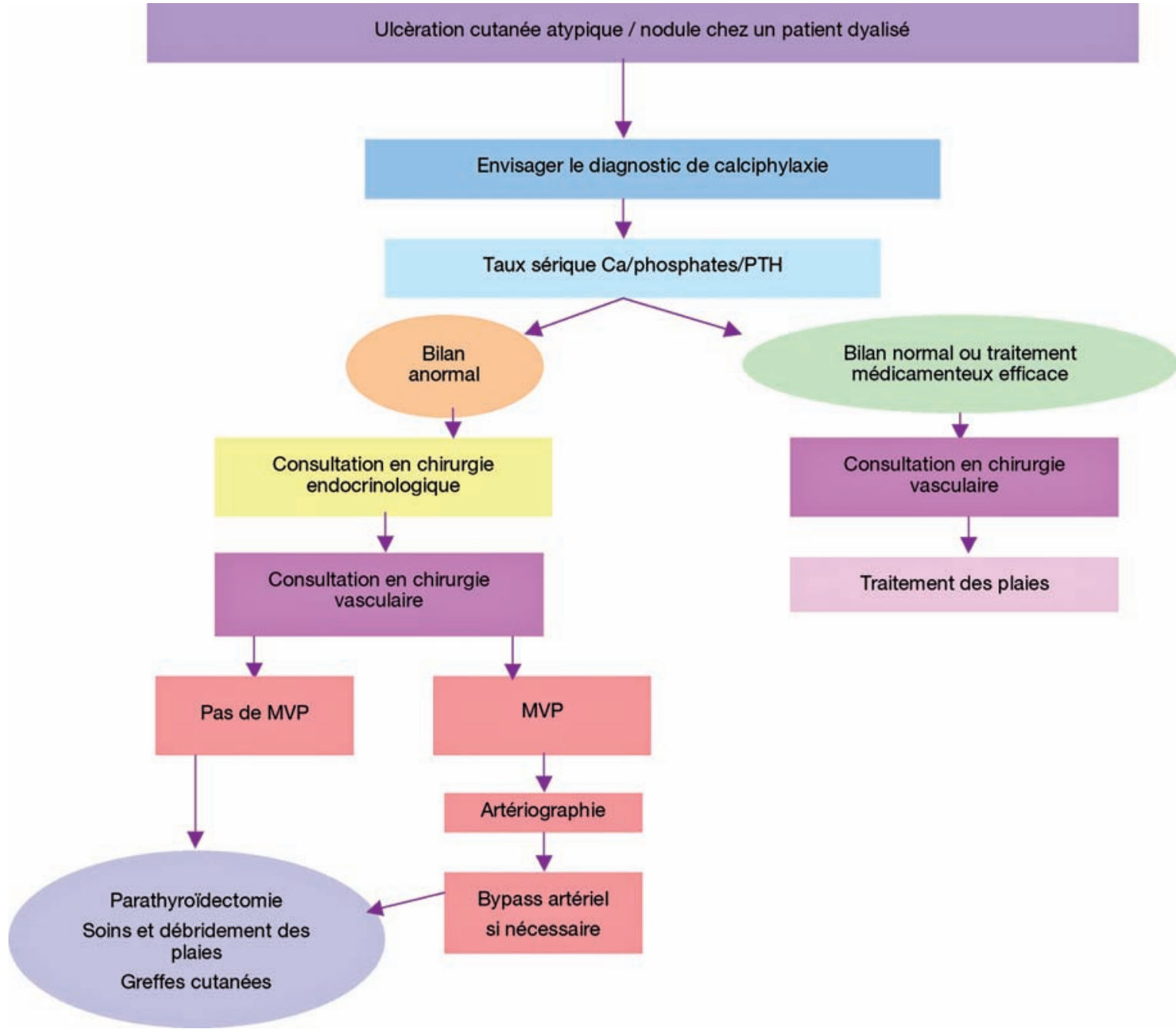

médecine buccale chirurgie buccale

VOL. $12, \mathrm{~N}^{\circ} 2$ 2006

page 97 


\section{CONCLUSION}

Pour résumer la présentation de la calciphylaxie, on peut, comme Kang et al. ${ }^{[29]}$, rappeler que :

- c'est une maladie rare avec une incidence de $1 \%$ chez des patients atteints d'IRC,et une prévalence de $4,1 \%$ chez des patients hémodyalisés,

- une prépondérance féminine avec une large tranche d'âge,

- son étiologie est probablement multifactorielle,

- les lésions doivent être régulièrement désinfectées et débridées,

- les infections contrôlées par une antibiothérapie adaptée,

- la recherche d'une autre maladie vasculaire périphérique devrait être systématiquement effectuée chez les patients atteints d'une IRC,

- les indications chirurgicales (amputation et parathyroïdectomie) sont réservées aux cas

médecine

buccale

chirurgie

buccale

VOL. $12, \mathrm{~N}^{\circ} 2$ 2006

page 98
Les manifestations de la calciphylaxie sont d'intensité variable et il existe probablement des formes mineures méconnues, à côté de celles se révélant par un tableau clinique majeur. Cette affection a donc une morbidité et une mortalité très variables et il n'existe aucun traitement étiologique. Exceptionnellement, les premières manifestations peuvent intéresser la région cervico-faciale. La présentation d'un cas est mise à profit pour rappeler cette éventualité et décrire avec précision les signes cliniques du stade initial de la maladie. L'apparition d'une claudication intermittente évolutive de la langue, compliquée d'une nécrose ischémique linguale trois mois plus tard, aurait dû d'emblée faire évoquer une calciphylaxie. Malheureusement la présence de séquelles d'une irradiation cervico-faciale et la calcification des branches terminales de l'artère faciale ont fait suspecter en premier lieu, respectivement des complications post-radiques ou une maladie de Horton.

\section{RÉFÉRENCES}

1 - Bedoya R M, Gutierrez J L, Mayorga F. Calciphylaxis causing localized tongue necrosis: a case report. J Oral Maxillofac Surg 1997 ; 55 : 193-6.

2 - Rudwaleit M, Schwarz A, Trautmann C, Offermann G, DISTLER A. Severe calciphylaxis in a renal patient on longterm oral anticoagulant therapy. Am J Nephrol 1996 ; $16: 344-8$.

3 - SMILEY C M, Hanlon S U, Michel D M. Calciphylaxis in moderate renal insufficiency: changing disease concepts. Am J Nephrol 2000 ; 20 : 324-8.

4 - SELYE H. Experimental calciphylaxis. Verh Dtsch Ges Inn Med $1965 ; 71:$ 504-10.

5 - Khafif R A, Delima C, Silverberg A, Frankel R. Calciphylaxis and systemic calcinosis. Collective review. Arch Intern Med 1990 ; 150 : 956-9.

6 - Ahmed S, O'Neill K D, Hood A F, Evan A P, Moe S M. Calciphylaxis is associated with hyperphosphatemia and increased osteopontin expression by vascular smooth muscle cells. Am J Kidney Dis 2001 ; 37 : 1267 76.

7 - BARgman J M. Calciphylaxis, calcinosis and calcergyseparate but not equal. J Rheumatol 1995 ; 22 : 5-7.

8 - ESSARY L R, WICK M R. Cutaneous calciphylaxis. An underrecognized clinicopathologic entity. Am J Clin Pathol $2000 ; 113: 280-7$
9 - Elamin E M, McDonald A B. Calcifying panniculitis with renal failure: a new management approach. Dermatology 1996 ; 192 : 156-9.

10 - Wilmer W A, MAgro C M. Calciphylaxis: emerging concepts in prevention, diagnosis, and treatment. Semin Dial $2002 ; 15: 172-86$.

11 - Podymow T, WherRetT C, Burns K D. Hyperbaric oxygen in the treatment of calciphylaxis: a case series. Nephrol Dial Transplant 2001; 16 : 2176-80.

12- Angelis $M$, Wong L L, Myers $S$ A, Wong L $M$. Calciphylaxis in patients on hemodialysis: a prevalence study. Surgery 1997; 122 : 1083-9 ; discussion 108990.

13 - Girotto J A, Harmon J W, Ratner L E, Nicol T L, WONG L, CHEN H. Parathyroidectomy promotes wound healing and prolongs survival in patients with calciphylaxis from secondary hyperparathyroidism. Surgery 2001; 130 : 645-50 ; discussion 650-1.

14 - BudisavlueVIc M N, CheEk D, Ploth D W. Calciphylaxis in chronic renal failure. J Am Soc Nephrol 1996; 7 : 978-82.

15 - LLACH F. The evolving pattern of calciphylaxis: therapeutic considerations. Nephrol Dial Transplant 2001; 16: 448-51. 
16 - Mathur R V, ShortLand J, Rel-Nahas A M. Calciphylaxis. Postgrad Med J $2001 ; 77$ : 557-61.

17 - Matsuo T, TSukamoto Y, Tamura M, Hanaoka M, NagaOKa T, KOBAYASHI Y, HIGaSHIHARA M, YOKOYAMa H, SAEGUSA N. Acute respiratory failure due to "pulmonary calciphylaxis" in a maintenance haemodialysis patient. Nephron 2001; 87 : 75-9.

18 - MathuR R V, ShORTLANd J R, El Nahas A M. Calciphylaxis with facial involvement. Nephrol Dial Transplant 2001 ; $16: 2256-7$.

19 - HafNer J, KeUsch G, Wahl C, BuRG G. Calciphylaxis: a syndrome of skin necrosis and acral gangrene in chronic renal failure. Vasa $1998 ; 27: 137-43$.

20 - FINE A, ZACHARIAS J. Calciphylaxis is usually non-ulcerating: risk factors, outcome and therapy. Kidney Int $2002 ; 61: 2210-17$.

21 - Bleyer A J, Choi M, Igwemezie B, de La Torre E, White W L. A case control study of proximal calciphylaxis. Am J Kidney Dis 1998 ; 32 : 376-83.

22 - Hafner J, Keusch G, Wahl C, Sauter B, Hurlimann A, von Weizsacker F, Krayenbuhl M, Biedermann K, BRUNNER U, HELFENSTEIN U. Uremic small-artery disease with medial calcification and intimal hyperplasia (so-called calciphylaxis): a complication of chronic renal failure and benefit from parathyroidectomy. J Am Acad Dermatol 1995 ; 33 : 954-62.

23 - Dereure O, Leray H, Barneon G, Canaud B, Mion C, GUILHOU J J. Extensive necrotizing livedo reticularis in a patient with chronic renal failure, hyperparathyroidism and coagulation disorder: regression after subtotal parathyroidectomy. Dermatology 1996 ; 192 : 167-70.
24 - Mazhar A R, Johnson R J, Gillen D, Stivelman J C, Ryan M J, Davis C L, SteHman-Breen C O. Risk factors and mortality associated with calciphylaxis in end-stage renal disease. Kidney Int 2001 ; 60 : 324-32.

25 - BarRi Y M, GRAVES G S, KnOChel J P. Calciphylaxis in a patient with Crohn's disease in the absence of endstage renal disease. Am J Kidney Dis 1997 ; 29 : 7736.

26 - Edelstein C L, Wickham M K, Kirby P A. Systemic calciphylaxis presenting as a painful, proximal myopathy. Postgrad Med J 1992 ; 68 : 209-11.

27 - Kane W J, Petty P M, Sterioff S, McCarthy J T,CROTTY T B. The uremic gangrene syndrome: improved healing in spontaneously forming wounds following subtotal parathyroidectomy. Plast Reconstr Surg 1996 ; $98: 671-678$.

28 - Pollock B, Cunliffe W, JMerchant W J. Calciphylaxis in the absence of renal failure. Clin Exp Dermatol 2000 ; $25: 389-92$.

29 - Kang A S, McCarthy J T, Rowland C, Farley D, Rvan HEERDEN J A. Is calciphylaxis best treated surgically or medically? Surgery 2000 ; 128 : 967-71; discussion 971-2.

30 - Milas M, Bush R L, LIN P, BROWN K, Mackay G, Lumsden A, WeBER C, DODSON T F. Calciphylaxis and nonhealing wounds: the role of the vascular surgeon in a multidisciplinary treatment. J Vasc Surg 2003 ; 37 : 501-7.

31 - Denton M D, Mauiryedi S, Bazari H. Heparin-induced skin necrosis in a patient with end-stage renal failure and functional protein S deficiency. Am J Nephrol 2001 ; $21: 289-93$. \begin{tabular}{l}
$\begin{array}{l}\text { médecine } \\
\text { buccale } \\
\text { chirurgie } \\
\text { buccale }\end{array}$ \\
\hline VOL. 12, N ${ }^{\circ} 2$ \\
2006
\end{tabular}

page 99 